\title{
SÍNTESE E CARACTERIZAÇÃO DE MOFs UTILIZANDO ÁCIDO 1,4- BENZENODICARBOXÍLICO E ZINCO: NOVA ROTA VERDE HIDROTÉRMICA
}

\author{
P. R. B. BRAZÃO ${ }^{1}$, T. DA S. ROCHA ${ }^{2}$, E. L.V. MENDONÇA ${ }^{3}$, A. M.U. ARAÚJO ${ }^{4}$, A. P. DE A. NASCIMENTO, ${ }^{5}$ E. \\ MOURA NETO', J. F. S. NASCIMENTO' \\ Instituto Federal de Educação, Ciência e Tecnologia do Rio Grande do Norte ${ }^{1-3,5-7}$, Universidade Federal do Rio \\ Grande do Norte 4 \\ jarley.nascimento@ifrn.edu.br ${ }^{7}$ \\ Submetido 01/04/2020 - Aceito 07/08/2020 \\ DOI: $10.15628 /$ holos.2020.9823
}

\section{RESUMO}

Neste estudo, foi reproduzida a síntese em triplicata, desenvolvida por Yaghi e seus colaboradores, na síntese da rede metal-orgânica conhecida como MOF-5. No entanto, não foi utilizada dimetilformamida (DMF) como solvente, sendo este solvente orgânico substituído por água destilada, para preparação da síntese hidrotérmica, fato até onde se sabe não relatado na literatura. Dessa forma, teríamos uma síntese dentro do âmbito da química verde, uma vez que, estaríamos evitando a produção de resíduos, utilização de um produto menos perigoso como solvente (toxicidade inócua), e não utilização de solventes oriundos de fontes não renováveis. A reprodutibilidade de síntese, dos pós obtidos foi confirmado a partir das técnicas de difração de raios-X (DRX) e espectroscopia de infravermelho com transformada de Fourier - refletância total atenuada (IVTF-RTA). O material obtido por essa rota verde hidrotérmica, denominada como MOF-5-RVH, apresentou padrões de difração diferentes da MOF-5 na sua fase pura e interpenetrada. Tal fato, pode ser explicado a partir da influência do solvente na cristalização da rede, que fez com que o processo de nucleação dos cristais não originasse a morfologia cúbica típica da MOF-5, que foi confirmado a partir das análises de microscopia eletrônica de varredura (MEV).

PALAVRAS-CHAVE: MOFs; Síntese; Ambiental; química verde; caracterização.

\section{SYNTHESIS AND CHARACTERIZATION OF MOFS USING 1,4-BENZENODICARBOXYLIC ACID AND ZINC: NEW HYDROTHERMAL GREEN ROUTE}

\begin{abstract}
In this study, the triplicate synthesis, developed by Yaghi and his collaborators, was reproduced in the synthesis of the metal-organic network known as MOF-5. However, dimethylformamide (DMF) was not used as a solvent, this organic solvent being replaced by distilled water, for the preparation of hydrothermal synthesis, a fact as far as is not reported in the literature. In this way, we would have a synthesis within the scope of green chemistry, since we would be avoiding the production of waste, using a less dangerous product as a solvent (harmless toxicity), and not using solvents from non-renewable sources. The reproducibility of the synthesis of the powders obtained
\end{abstract}

was confirmed using the techniques of X-ray diffraction (XRD) and infrared spectroscopy with Fourier transform attenuated total reflectance (FTIR-ATR). The material obtained by this green hydrothermal route, called MOF$5-\mathrm{RVH}$, showed diffraction patterns different from MOF5 in its pure and interpenetrated phase. This fact can be explained by the influence of the solvent on the crystallization of the network, which made the process of nucleation of the crystals not originate the cubic morphology typical of MOF-5, which was confirmed from the scanning electron microscopy analyzes (MEV).

KEYWORDS: MOFs; synthesis; environmental; green chemistry; characterization. 


\section{INTRODUÇÃO}

As redes metalorgânicas (MOFs, do inglês - Metal-organic framework) se caracterizam por apresentar grande estabilidade, baixa densidade, alta porosidade, alta área superficial específica e estruturas cristalinas bem definidas (Batten et al., 2013). Classificadas como materiais híbridos inorgânico-orgânicos, formadas a partir da interação de íons metálicos ou clusters e ligantes orgânicos politópicos (Yaghi et al., 2003). Para se obter as MOFs, normalmente utiliza-se ligantes multidentados que possuam átomos doadores de elétrons (Nascimento et al., 2017).

Diversos ligantes têm sido desenvolvidos e aplicados em novas sínteses, dentre as quais se destaca os policarboxilatos aromáticos, devido a sua robustez química (Janiak e Vieth, 2010). O ácido tereftálico $\left(1,4-\mathrm{H}_{2} \mathrm{BDC}\right)$ apresenta características propícias na construção das MOFs, atuando como ponte entre os centros metálicos, podendo acarretar estruturas uni, bi ou tridimensionais (1D, 2D e 3D) (Ramos, Tanase \& Rothenberg, 2014).

Essas redes também chamadas polímeros de coordenação vem se destacando no âmbito das pesquisas por apresentarem tamanho elevados dos poros, assim como podem proporcionar a adsorção de maiores moléculas e consequentemente possibilitar a ampliação dos processos catalíticos (Sharma \& Feng, 2019). Diante dessa perspectiva, e devido as suas propriedades específicas, estudos vêm sendo desenvolvidos utilizando tais materiais para aplicações em diversas áreas, como armazenamento de gases (Frem et al., 2018), fotônica (Cui, He \& Qian, 2018), sensoriamento de moléculas (Zhang et al., 2018), incorporação e carreamento de fármacos (Lima Neto et al., 2019) entre outros.

Visto a importância estratégica desses materiais, iremos reproduzir a síntese em triplicata, desenvolvida por Yaghi e seus colaboradores (1999), na síntese da rede metal-orgânica conhecida como MOF-5. No entanto, não foi utilizada dimetilformamida (DMF) como solvente, sendo este solvente orgânico substituído por água destilada, para preparação da síntese hidrotérmica, fato até onde se sabe não relatado na literatura. Dessa forma, teríamos uma síntese dentro do âmbito da química verde, uma vez que, estaríamos evitando a produção de resíduos, utilização de um produto menos perigoso como solvente (toxicidade inócua), e não utilização de solventes oriundos de fontes não renováveis (Lenardão et al., 2003).

Os pós obtidos nas sínteses a partir dessa rota verde, foram caracterizados por, difração de raios-X (DRX), espectroscopia de infravermelho com transformada de Fourier - refletância total atenuada (IVTF-RTA), e microscopia eletrônica de varredura (MEV).

\section{REVISÃO BIBLIOGRÁFICA}

\subsection{Breve histórico das redes metal-orgânicas}

Polímeros de coordenação, termo utilizado desde o ano de 1954, quando Wells (Wells, 1954) introduziu o conceito de estruturas cristalinas inorgânicas, onde os íons metálicos, agindo como nós, ligam-se entre si através de um espaçador (spacer), tais como as moléculas multidentadas dos polímeros de coordenação. A topologia da rede resultante, depende tanto da 
geometria quanto do ambiente de coordenação dos íons metálicos (nós), sendo o espaçador apenas uma amarração linear entre os nós adjacentes (Wiley \& Weinheim, 2011).

Um outro trabalho de suma importância foi desenvolvido por Kinoshita (Kinoshita et al., 1959), em seus estudos desenvolveu-se um trabalho sobre polímeros de coordenação cristalinos, trabalho esse que foi considerado um dos pioneiros, onde foi descrito a estrutura do composto de cobre, que resultou em uma rede polimérica parecida com diamante. Já em 1989, partindo dos estudos de Hoskins e Robson (1989), sobre redes poliméricas constituídas por seguimentos tridimensionais, ou seja, elementos que possuem três dimensões, com unidades conexão haste que foram classificados pelos autores como uma nova "redescoberta". Ainda neste estudo, os autores mencionaram o potencial destes materiais em catálise, entretanto, não foi realizado nenhum estudo com embasamento experimental. Somente em 1994, surgiu a possibilidade de aplicar essa classe de polímeros em reações catalítica, na reação de cianosililação de aldeídos (Fujita \& Maki, 1994).

Em 1995 o termo "Metal-organic framework", surgiu na literatura de periódicos nos trabalhos de Yaghi e Li (1995). Vale ressaltar que nenhum dos estudos que tinham um embasamento experimental, pois, não citavam dados de porosidade para aplicações catalíticas. Um grande avanço nos estudos dos polímeros de coordenação, ocorreu quando iniciou-se o desenvolvimento de materiais com elevada porosidade, estáveis termicamente e quimicamente, e sem a presença de moléculas visitantes (Ramos, Tanase \& Rothenberg, 2014).

\subsection{Redes metalorgânicas: nomenclaturas e definições}

Na Química de coordenação, os metais apresentam diversos números de coordenação, tendo como uma das consequências, a adoção de diversas formas geométricas por seus compostos (Leite, 2014).

$\mathrm{Na}$ formação das ligações coordenadas entre o ligante orgânico e os centros metálicos, pode ocorrer a coordenação de outras moléculas, tendo como exemplo, $\mathrm{CO}_{2}, \mathrm{H}_{2} \mathrm{O}, \mathrm{Cl}$, etc. $\mathrm{O}$ próprio centro metálico, pode apresentar algumas possibilidades de geometrias, as chamadas unidades secundarias de construção (secondary building units - SBU), que representam forma a qual o metal está coordenado (Ramos, Tanase e Rothenberg, 2014). As SBUs, são complexos e agrupamentos, aos quais os modos de coordenação podem ser usados para a transformação destes fragmentos em redes porosas, utilizando os ligantes politópicos de forma repetitiva, assim podendo originar estruturas em duas ou três dimensões (Ramos, Tanase e Rothenberg, 2014). A SBU é composta por apenas um centro metálico, e um bloco de construção molecular conhecido como, MMB (single-metal-ion-based molecular building block) (Ramos, Tanase \& Rothenberg, 2014).

Em outros, ocorre a formação de agregados atômicos de ácidos metálicos, os chamados clusters. Uma das mais conhecidas é a chamada haste-roda (paddle-wheel) (Ramos, Tanase \& Rothenberg, 2014). Ela é formada por dois átomos metálicos, que se ligam em ponte com quatro grupos carboxilatos (Perry, Perman \& Zaworotko, 2009).

Em relação ao ligante orgânico, há a possibilidade de existência de uma diversidade de compostos que podem ser utilizados, sendo um dos mais comuns o ácido tereftálico (Ramos, 
Tanase \& Rothenberg, 2014). O modo como os grupos de coordenação estão posicionados na molécula do ligante, é essencial para a definição estrutural final da MOF, sendo decisivo a escolha do ligante, para obtenção das propriedades desejadas na MOF (Perry, Perman \& Zaworotko, 2009).

A composição das MOFs é caracterizada pela presença de células unitárias, partindo da união das duas SBUs, formando uma rede tridimensional que caracteriza a porosidade e cristalinidade da MOF. As características geométricas e químicas das unidades secundárias de construção, dão à previsão da topologia da rede (Paz et al., 2012). Tal como a MOF-5, que é formada por uma célula unitária de formato cúbico, sendo que em cada vértice está presente um agrupamento do tipo $\mathrm{Zn}_{4}(O) O_{12} C_{6}$, se ligando através de uma molécula do ligante 1,4-BDC, compostas nas arestas do cubo, estrutura conhecida como PCU (primitive cubic unit) (Paz et al., 2012). A cavidade interna da MOF-5, é capaz de hospedar uma esfera de 18,5 $\AA$ (Figura 1). Os 4 clusters $\mathrm{ZnO}_{4}$ com um vértice comum, unidos entre si por 6 grupos carboxilatos, originam uma SBU octaédrica.
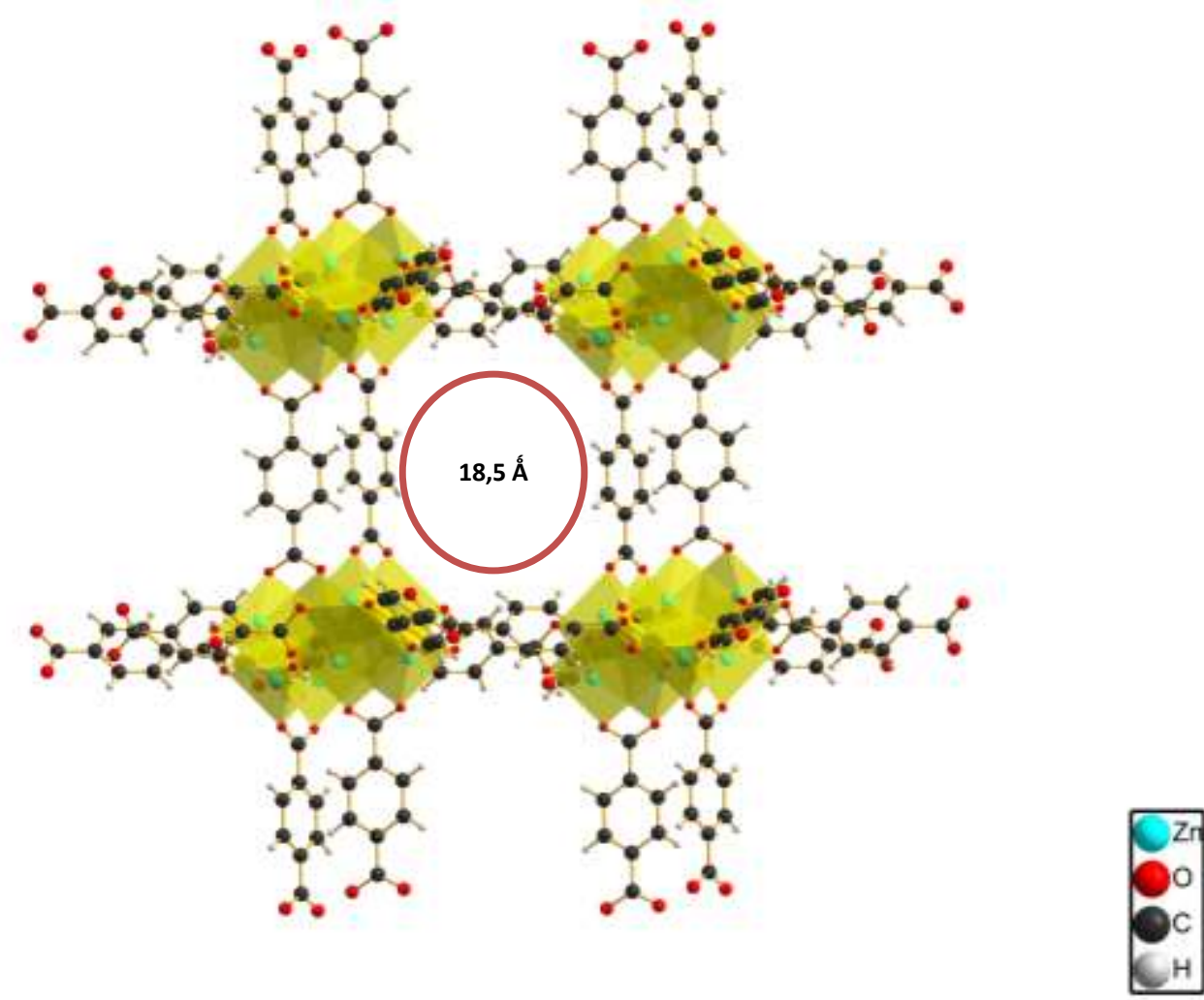

Figura 1: Representação da MOF-5 parcialmente estendida para demonstrar seu volume de poro (Os tetraedros em amarelo representam os Clusters do $\mathrm{ZnO}_{4}$ ).

Em relação a nomenclatura desta classe de materiais, o tempo relativamente curto de descoberta dos mesmos, e o fato de ser um campo de pesquisa multidisciplinar, que envolve diversas áreas da química (química do estado sólido, inorgânica e de coordenação), faz com que ainda não haja uma nomenclatura padrão (Ramos, Tanase \& Rothenberg, 2014), ocorrendo, uma interposição de conceitos, terminologias e classificações (Farrusseng, Aguado \& Pinel, 2009), tendo como exemplo, os termos "polímeros de coordenação" e "redes metal-orgânicas" que em muitos dos casos são considerados sinônimos, sendo o último um subgrupo do primeiro (Farrusseng, 
Aguado e Pinel, 2009). Alguns autores julgam que, para ser classificado como MOF, deve ocorrer a investigação se o material é poroso por meio de isotermas de adsorção. Outros julgam, que basta o material ter uma estrutura 2-D ou 3-D com ligantes orgânicos (Ramos, Tanase \& Rothenberg, 2014)

Segundo Rowsell e Yaghi (2006), é impróprio o material ser classificado como MOF, se este foi caracterizado apenas por cristalografia, uma vez que seria necessário, que a molécula do solvente seja eliminada ou trocada, sem a perda de integridade da MOF. Para esse fim, o uso de ligantes multidentados é necessário, com o intuito de que se forme uma estrutura rígida com ligações do tipo M-O-C (Kitagawa \& Kondo, 1998). Estes autores, reportaram a classificação dos polímeros de coordenação em gerações: onde a primeira seria composta por aqueles em que a rede porosa seria amparada por moléculas vizinhas, a qual colapsaria com a remoção das mesmas; a segunda seria formada por sistemas porosos robustos, onde a porosidade seria permanente, ainda que ocorresse a retirada de moléculas de solvente (as primeiras MOFs sintetizadas, estariam classificadas nesta geração); e a terceira, com sistema poroso flexível, com a probabilidade de mudanças reversíveis dependendo da presença de moléculas visitantes ou em resposta a estímulos externos, tais como luz, temperatura ou campos elétrico e magnético.

Em 2009 a IUPAC iniciou um projeto de unificação de nomenclatura, intitulado "Coordination polymers and metal-organic frameworks: terminology and nomenclature guidelines", encontra-se uma recomendação provisória publicada, porém, ainda não concluída (Batten et al., 2013). No documento a definição de MOF como polímero de coordenação (alternativamente rede de coordenação), é caracterizado por ser uma rede aberta (open framework) contendo espaços potenciais (Frem et al., 2018). Observa-se que a IUPAC caminha para uma definição genérica, deixando claro que o espaço interno precisa estar disponível e as moléculas do solvente devem estar livres para fluir e sua remoção ser testada (Frem et al., 2018). O documento também faz a recomendação do uso de topologia para a melhor descrição das estruturas dos cristais das MOFs e dos polímeros de coordenação 3-D, segundo descrito no trabalho de (O'Keeffe et al., 2008).

As MOFs têm sido denominada atualmente com uma abreviação, seguido de um número sequenciado em termos de descoberta. As abreviações podem indicar o tipo de material (Ex: MOF metal organic framework) o tipo de estrutura (Ex: ZMOF=MOF com estrutura similar à uma zeólita) ou o laboratório que o material foi preparado (Ex: CPO = coordination polymer of Oslo) (Frem et al., 2018). O uso da fórmula empírica é uma possibilidade a esta nomenclatura, expondo o(s) metal(is), o(s) ligante(s) e a estequiometria na célula unitária, podendo aparecer também a molécula "visitante", Ex: $[\mathrm{Zn} 4(\mathrm{O})(\mathrm{bdc}) 3] .3 \mathrm{H}_{2} \mathrm{O}$, sendo que o oxigênio e a molécula de 1,4benzenodicarboxilato (BDC) são ligantes e a $\mathrm{H}_{2} \mathrm{O}$ é uma molécula "visitante" (Frem et al., 2018).

\section{METODOLOGIA}

Os reagentes utilizados no processo de síntese das redes metalorgânicas encontram-se listados na Tabela 1. Estes tem seu grau de pureza atestado pelos respectivos fabricantes e não passaram por nenhuma purificação prévia adicional. 
Tabela 1: Reagentes utilizados na síntese

\begin{tabular}{ccc}
\hline Reagentes (grau de pureza) & Fórmula Química & Fabricante \\
\hline Ácido tereftálico (98\%) & $\mathrm{C}_{6} \mathrm{H}_{4}(\mathrm{COOH})_{2}$ & Aldrich \\
Nitrato de Zinco Hexaidratado & $\mathrm{Zn}\left(\mathrm{NO}_{3}\right)_{2} .6 \mathrm{H}_{2} \mathrm{O}$ & Aldrich \\
$(99,7 \%)$ & & \\
& $\mathrm{CH}_{3} \mathrm{CH}_{2} \mathrm{OH}$ & Vetec \\
Álcool etílico P.A $(99,8 \%)$ & $\mathrm{HNO}_{3}$ & Vetec \\
Ácido nítrico P.A. (69\%) & & \\
\hline
\end{tabular}

\subsection{Síntese da MOF}

Seguiremos o método desenvolvido por Yaghi e seus colaboradores (Eddaoudi, O'Keeffe e Yaghi, 1999), na obtenção da MOF-5. No entanto, estamos propondo um rota verde sem a utilização de solventes orgânicos, que serão substituídos por água destilada obedecendo aos princípios da Química verde. Neste processo, Foram pesados $2 \mathrm{mmol}$ de $\mathrm{Zn}\left(\mathrm{NO}_{3}\right)_{2} \cdot 6 \mathrm{H}_{2} \mathrm{O}$ e $1 \mathrm{mmol}$ de tereftalato de sódio (preparado previamente, através da solubilização do ligante em $15 \mathrm{ml}$ de água via adição de 5 gotas de $\mathrm{NaOH} 1 \mathrm{~mol} / \mathrm{L}$ ), a mistura reacional foi adicionada a um béquer de 25 $\mathrm{ml}$, e mantido sob agitação constante durante 15 minutos. Em seguida a solução resultante foi transferida para um reator de teflon com "camisa" de aço, e levado para uma estufa a $140^{\circ} \mathrm{C}$ por 12 horas.

Os sólidos obtidos foram lavados em triplicata com etanol e centrifugados por 60 segundos a $3600 \mathrm{rpm}$. Os pós obtidos foram secos por 24 horas na estufa a $70^{\circ} \mathrm{C}$ e posteriormente caracterizados.

\subsection{Caracterização da MOF}

A MOF foi caracterizada a partir das técnicas de, Espectroscopia de infravermelho com transformada de Fourier (IVTF-RTA), Difração de Raios-X (DRX) e Microscopia eletrônica de varredura (MEV).

Os espectros de infravermelho por transformada de Fourier (IVTF-RTA), foi obtido com o espectrômetro Bruker IVTF - Vertex 70, com platinun RTA, analisando o pó do material em uma faixa entre 4000-400 $\mathrm{cm}^{-1}$ ). As medidas de difração de raios-X, foram feitas em um difratômetro Bruker D2 Phaser, utilizando radiação Cuka ( $\lambda=1,5406$ Á) contendo filtro de níquel. A faixa de obtenção do difratograma foi entre $3^{\circ}$ e $70^{\circ}$. A morfologia dos pós, foi analisada utilizando um microscópio $\mathrm{FEI}$ - Inspect F50, com tensão de aceleração de 5 kV, modelo TM 3000.

\section{RESULTADOS E DISCUSSÃO}

Para verificar se ocorreu a síntese da MOF, os pós das amostras obtidos em triplicata utilizando uma razão molar de 2:1 metal-ligante, foram caracterizados via DRX, IVTF-RTA e MEV, sendo estas discursões abordadas nas Figuras 2, 3 e 4 respectivamente 
A MOF-5-RVH (O RVH significa rota verde hidrotérmica), apresenta picos com intensidades e posições distintas do ligante puro $1,4-\mathrm{H}_{2} \mathrm{BDC}$, o que sugere que a síntese ocorreu de forma satisfatória (Figura 2). A MOF-5 monofásica obtida por Yaghi e seus colaboradores (Eddaoudi, O'Keeffe e Yaghi, 1999), apresenta como principais picos as posições $6.8^{\circ}, 9^{\circ} 6^{\circ}, 13.7^{\circ}$ e $15.4^{\circ}$ em ordem decrescente de intensidade. Quando comparamos com o nosso material MOF-5-RVH, este não apresenta os picos e as intensidades características do padrão calculado da MOF-5, a partir dos dados do CCDC (Cambridge Crystalographic data centre).

Segundo Campos (2014), e após analisar o banco de dados de North Western, observou-se que a MOF-5 pode ter mais dois padrões de difração já catalogados além da fase pura. $O$ primeiro trata deste material apresentando uma interpenetração, o que pode ser identificado a partir de picos intensos em $9,6^{\circ}$ e $13,7^{\circ}$, que não são observados nos difratogramas da MOF-5-RVH. 0 segundo trata do padrão de difração da MOF-5 com duas interpenetrações, que pode ser identificado a partir de um pico intenso em $9,6^{\circ}$ e picos com baixa intensidade em $12.1^{\circ}$ e $15.4^{\circ}$. Os últimos dois picos com baixa intensidade da estrutura com duas interpenetrações, são observados nos padrões de difrações um pouco deslocados em $12.2^{\circ}$ e $15.6^{\circ}$ (Figura 2). Contudo, a MOF-5-RVH, não apresenta os picos mais intensos característicos dos três padrões de difração possíveis, o que sugere que a morfologia dos cristais formados do material sintetizado, não deve refletir a clássica morfologia cúbica da MOF-5, o que poderia ser justificado pela influência do solvente no processo de nucleação dos cristais.

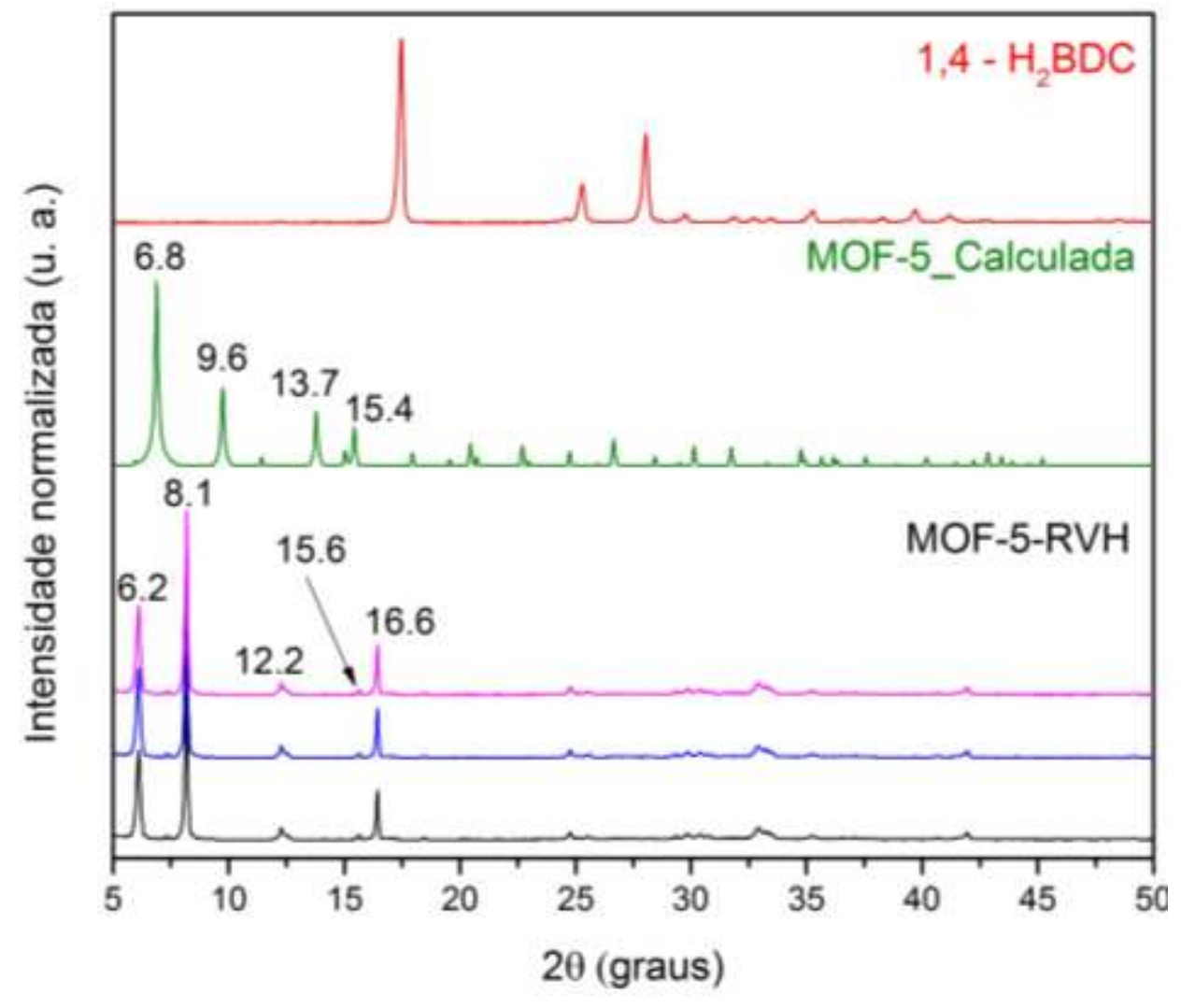

Figura 2: Comparação entre os difratogramas da MOF-5-RVH e ligante puro.

Os espectros de infravermelho das três amostras sintetizadas (MOF-5-RVH) são bastante semelhantes, e devido à baixa simetria dos íons carboxilatos (RCOO-), diferentes modos de 
coordenação podem ser distinguidos com base no número de vibrações ativas na região do infravermelho (Figura 2). A diferença entre as vibrações referentes a estiramentos assimétricos $\mathrm{U}_{\mathrm{as}}\left(\mathrm{COO}^{-}\right)$e simétricos $\mathrm{U}_{\mathrm{s}}\left(\mathrm{COO}^{-}\right)$fornece informação relevante sobre a natureza dos modos de coordenação do íon carboxilato (Deacon e Philips, 1980). A ausência da banda do ligante em 1672 $\mathrm{cm}^{-1}$ nas amostras MOF-5-RVH referente a vibração da carbonila $(\mathrm{C}=\mathrm{O})$ no grupo carboxila $(\mathrm{COOH})$, confirma a coordenação dos centros metálicos e demonstra que todo o ligante foi consumido. No espectro das amostras MOF-5-RVH, as bandas referentes a $v_{\text {as }}\left(C^{-} O^{-}\right)$em torno de $1571 \mathrm{~cm}^{-1}$, e $v_{s}$ (COO) em $1349 \mathrm{~cm}^{-1}$, podem ser observadas. A diferença $\Delta v=222 \mathrm{~cm}^{-1}$, revela que ambos os grupos $\mathrm{COO}^{-}$do 1,4-BDC estão coordenados ao $\mathrm{Zn}^{2+}$ de modo monodentado (Deacon e Philips, 1980). As absorções que ocorrem na faixa de $1264-959 \mathrm{~cm}^{-1}$ e de $797-653 \mathrm{~cm}^{-1}$ estão relacionadas com as vibrações das ligações $\mathrm{C}-\mathrm{H}$ do anel benzênico do ligante, no plano e fora do plano respectivamente, sendo observadas em todos os espectros mesmo após a coordenação do centro metálico (Canevarolo, 2004). A banda de absorção centrada em $3381 \mathrm{~cm}^{-1}$ para as amostras, é observada devido a coordenação de moléculas de água na estrutura da MOF-5-RVH.

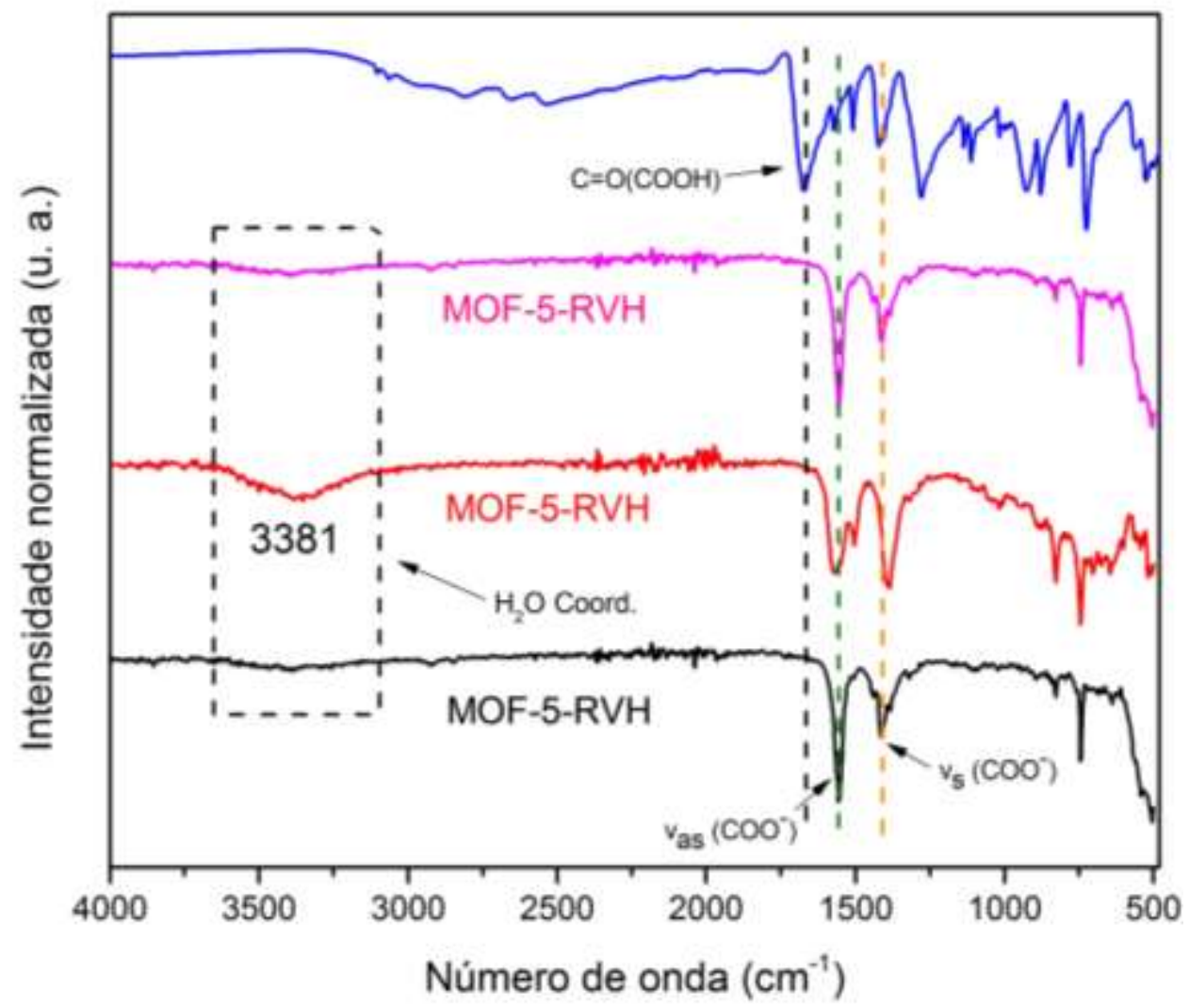

Figura 3: Comparação dos espectros de MOF-5-RVH, com o 1,4- $\mathrm{H}_{2} \mathrm{BDC}$ puro.

Um dos fatores que influenciam diretamente no processo de formação de cristais de uma MOF, é a natureza do solvente utilizado (Frem et al., 2018). Utilizamos a água como solvente ao invés de solventes orgânicos já relatados na literatura (Li et al., 2015), mantendo todos os outros fatores que tem influência na morfologia destes materiais constantes. Observou-se para a MOF-5RVH uma mistura de fases, já que foi identificado uma morfologia majoritária em forma de bastões (destaque em vermelho) e outra em forma de agulhas (destaque em verde). Tal fato, corrobora os resultados de DRX com relação a não existência de microestruturas em forma de cubos. 

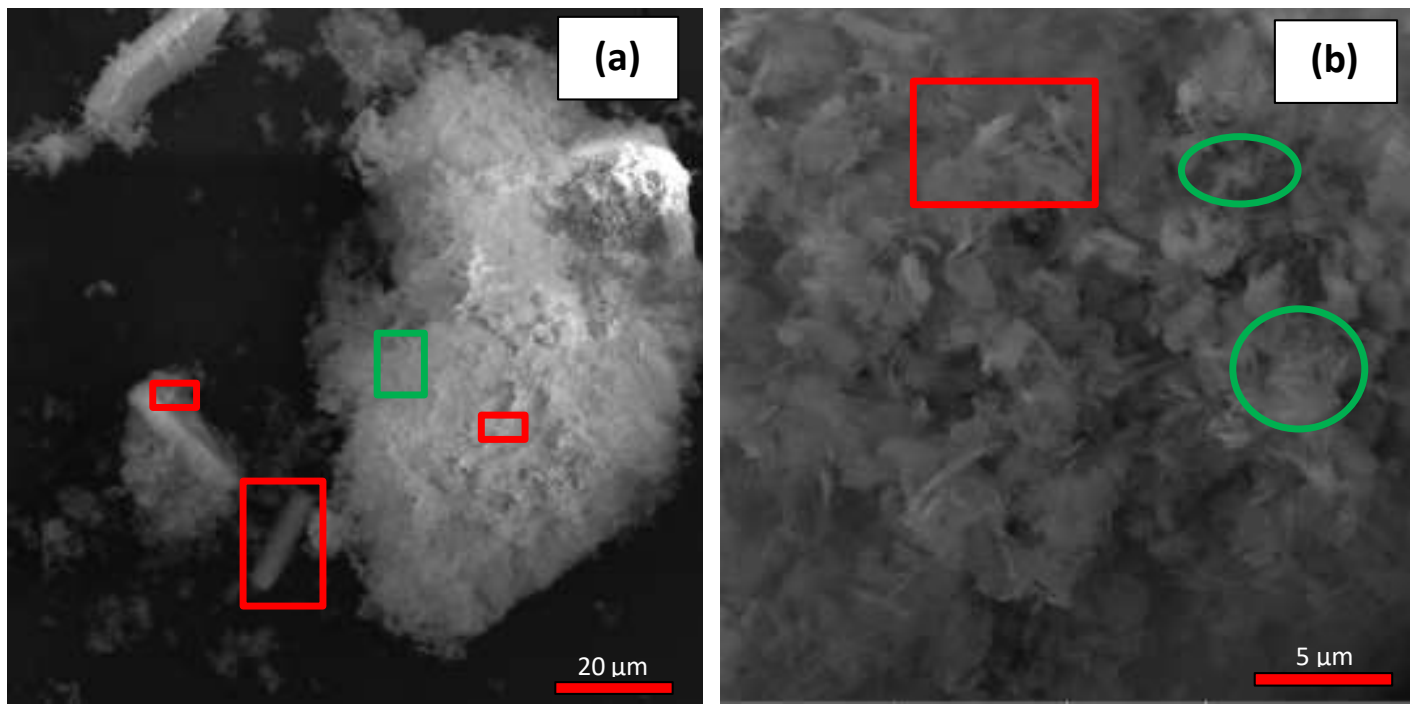

Figura 4: Microscopia eletrônica de varredura (MEV) da MOF-5-RVH (a) ampliado 2000x (b) ampliado 10000x.

\section{CONCLUSÃO}

Neste estudo, demonstramos uma rota verde para a MOF-5 a qual denominamos MOF-5RVH, que apresentou boa reprodutibilidade de síntese. Com base no infravermelho, foi possível determinar que ambos os íons COO- estão coordenados ao cluster no modo monodentado. Os padrões de difração das amostras, mostram que a rota utilizando apenas água, teve forte influência na formação da estrutura da MOF-5-RVH, promovendo uma nucleação dos cristais distinta da que estava reportada na literatura para a MOF-5, o que foi observado a partir dos padrões de difração. A morfologia apresentada pela MOF-5-RVH, também foi distinta da MOF-5, o que corrobora os resultados de DRX. Ambientalmente, a obtenção de uma rede metal-orgânica utilizando-se apenas água como solvente é extremamente vantajoso. No entanto, A MOF-5-RVH necessita em trabalhos futuros, ter sua porosidade e capacidade de estocagem de gases testadas, para podermos afirmar com maior exatidão, que a mesma é capaz de atuar de forma complementar ou substitutiva, perante uma rede metal-orgânica tão consolidada como a MOF-5, projetada por Yaghi e seus colaboradores.

\section{REFERÊNCIAS}

Batten, S. R., Champness, N. R., Chen, X. M., Garcia-Martinez, J., Kitagawa, S., Öhrström, L., ... \& Reedijk, J. (2013). Terminology of metal-organic frameworks and coordination polymers (IUPAC Recommendations 2013). Pure and Applied Chemistry, 85(8), 1715-1724.

Campos, V. D. O. (2014). Síntese e caracterização de redes metalorgânicas baseadas em zinco e ácidos benzenodicarboxílicos (Master's thesis, Universidade Federal do Rio Grande do Norte).

Canevarolo Jr, S. V. (2004). Técnicas de caracterização de polímeros. Artliber, São Paulo, 430(2004).

De Lima Neto, O. J., de Oliveira Frós, A. C., Barros, B. S., de Farias Monteiro, A. F., \& Kulesza, J. (2019). Rapid and efficient electrochemical synthesis of a zinc-based nano-MOF for Ibuprofen adsorption. New Journal of Chemistry, 43(14), 5518-5524. 
Deacon, G. B., \& Phillips, R. J. (1980). Relationships between the carbon-oxygen stretching frequencies of carboxylato complexes and the type of carboxylate coordination. Coordination Chemistry Reviews, 33(3), 227-250.

Farrusseng, D., Aguado, S., \& Pinel, C. (2009). Metal-organic frameworks: opportunities for catalysis. Angewandte Chemie International Edition, 48(41), 7502-7513.

Frameworks, M. O. (2011). Applications from catalysis to Gas Storage. Wiley-VCH Verlag GmbH \& Co. KGaA, Weinheim, Germany.

Frem, R. C., Arroyos, G., Flor, J. B., Alves, R. C., Lucena, G. N., Silva, C. M. D., \& Coura, M. F. (2018). MOFS (METAL-ORGANIC FRAMEWORKS): UMA FASCINANTE CLASSE DE MATERIAIS INORGÂNICOS POROSOS. Química Nova, 41(10), 1178-1191.

Fujita, N., \& Maki, T. (1994). U.S. Patent No. 5,306,845. Washington, DC: U.S. Patent and Trademark Office.

Hoskins, B. F., \& Robson, R. (1989). Infinite polymeric frameworks consisting of three dimensionally linked rod-like segments. Journal of the American Chemical Society, 111(15), 5962-5964.

Janiak, C., \& Vieth, J. K. (2010). MOFs, MILs and more: concepts, properties and applications for porous coordination networks (PCNs). New Journal of Chemistry, 34(11), 2366-2388.

Kitagawa, S., \& Kondo, M. (1998). Functional micropore chemistry of crystalline metal complexassembled compounds. Bulletin of the Chemical Society of Japan, 71(8), 1739-1753.

Leite, A. K. P. (2014). Síntese, estrutura e propriedades de polímeros de coordenação à base de íons lantanídeos e ácidos benzenodicarboxílicos (Master's thesis, Universidade Federal do Rio Grande do Norte).

Lenardão, E. J., Freitag, R. A., Dabdoub, M. J., Batista, A. C. F., \& Silveira, C. D. C. (2003). " Green chemistry": os 12 princípios da química verde e sua inserção nas atividades de ensino e pesquisa. Química Nova, 26(1), 123-129.

Li, B., Wen, H. M., Wang, H., Wu, H., Yildirim, T., Zhou, W., \& Chen, B. (2015). Porous metal-organic frameworks with Lewis basic nitrogen sites for high-capacity methane storage. Energy \& Environmental Science, 8(8), 2504-2511.

Li, H., Eddaoudi, M., O'Keeffe, M., \& Yaghi, O. M. (1999). Design and synthesis of an exceptionally stable and highly porous metal-organic framework. nature, 402(6759), 276-279.

Nascimento, J. F. S., Barros, B. S., Kulesza, J., de Oliveira, J. B. L., Leite, A. K. P., \& de Oliveira, R. S. (2017). Influence of synthesis time on the microstructure and photophysical properties of GdMOFs doped with Eu3+. Materials Chemistry and Physics, 190, 166-174.

O’Keeffe, M., Peskov, M. A., Ramsden, S. J., \& Yaghi, O. M. (2008). The reticular chemistry structure resource (RCSR) database of, and symbols for, crystal nets. Accounts of chemical research, 41(12), 1782-1789. 
Paz, F. A. A., Klinowski, J., Vilela, S. M., Tome, J. P., Cavaleiro, J. A., \& Rocha, J. (2012). Ligand design for functional metal-organic frameworks. Chemical Society Reviews, 41(3), 1088-1110.

Perry, I. (2009). JJ, Perman, JA \& Zaworotko, MJ Design and synthesis of metal-organic frameworks using metal-organic polyhedra as supermolecular building blocks. Chem. Soc. Rev, 38, 14001417.

Ramos, A. L. D., TANASE, S., \& ROTHENBERG, G. (2014). Redes metalorgânicas e suas aplicações em catálise. Quim. Nova, 123-133.

Rowsell, J. L., \& Yaghi, O. M. (2006). Effects of functionalization, catenation, and variation of the metal oxide and organic linking units on the low-pressure hydrogen adsorption properties of metal- organic frameworks. Journal of the American Chemical Society, 128(4), 1304-1315.

Sharma, V. K., \& Feng, M. (2019). Water depollution using metal-organic frameworks-catalyzed advanced oxidation processes: a review. Journal of hazardous materials, 372, 3-16.

Wells, A. F. (1954). The geometrical basis of crystal chemistry. Part 3. Acta Crystallographica, 7(12), 842-848.

Yaghi, O. M., \& Li, H. (1995). Hydrothermal synthesis of a metal-organic framework containing large rectangular channels. Journal of the American Chemical Society, 117(41), 10401-10402.

Yaghi, O. M., O'Keeffe, M., Ockwig, N. W., Chae, H. K., Eddaoudi, M., \& Kim, J. (2003). Reticular synthesis and the design of new materials. Nature, 423(6941), 705-714.

Zhang, Y., Yuan, S., Day, G., Wang, X., Yang, X., \& Zhou, H. C. (2018). Luminescent sensors based on metal-organic frameworks. Coordination Chemistry Reviews, 354, 28-45. 